\title{
Global Control for Partial Deduction through Characteristic Atoms and Global Trees
}

\author{
Michael Leuschel ${ }^{\star}$ and Bern Martens ${ }^{\star \star}$ \\ Department of Computer Science \\ Katholieke Universiteit Leuven \\ Celestijnenlaan 200A, B-3001 Heverlee, Belgium \\ e-mail : \{michael,bern $\} @$ cs.kuleuven.ac.be
}

\begin{abstract}
Recently, considerable advances have been made in the (online) control of logic program specialisation. A clear conceptual distinction has been established between local and global control and on both levels concrete strategies as well as general frameworks have been proposed. For global control in particular, recent work has developed concrete techniques based on the preservation of characteristic trees (limited, however, by a given, arbitrary depth bound) to obtain a very precise control of polyvariance. On the other hand, the concept of an m-tree has been introduced as a refined way to trace "relationships" of partially deduced atoms, thus serving as the basis for a general framework within which global termination of partial deduction can be ensured in a non ad hoc way.

Blending both, formerly separate, contributions, in this paper, we present an elegant and sophisticated technique to globally control partial deduction of normal logic programs. Leaving unspecified the specific local control one may wish to plug in, we develop a concrete global control strategy combining the use of characteristic atoms and trees with global (m-)trees. We thus obtain partial deduction that always terminates in an elegant, non ad hoc way, while providing excellent specialisation as well as fine-grained (but reasonable) polyvariance.

We conjecture that a similar approach may contribute to improve upon current (on-line) control strategies for functional program transformation methods such as (positive) supercompilation.
\end{abstract}

\section{Introduction}

A major concern in the specialisation of functional $([4,13,31])$ as well as logic programs $([22,15,8,28,5])$ has been the issue of control: How can the transformation process be guided in such a way that termination is guaranteed and results are satisfactory?

This problem has been tackled from two (until now) largely separate angles: the so-called off-line versus on-line approaches. Partial evaluation of functional

\footnotetext{
* Supported by the Belgian GOA "Non-Standard Applications of Abstract Interpretation".

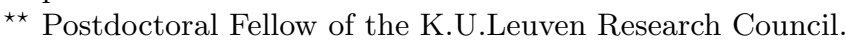


programs $([4,13])$ has mainly stressed the former, while supercompilation of functional $([31,32,30])$ and partial deduction of logic programs $([10,29,2,3,23$, $26,17]$ ) have concentrated on on-line control. (Some exceptions are $[33,27,16$, 14].) It is within this on-line control tradition that the present work provides a novel and important contribution.

In partial deduction of logic programs, one distinguishes two levels of control $([8,26])$ : the local and the global level. In a nutshell, the local level decides on how SLD(NF)-trees for individual atoms should be built. The (leaves of the) resulting trees allow to construct specialised clauses for the given atoms ([22, $1]$ ). At the global level on the other hand, one typically attends to the overall correctness of the resulting program (satisfying the closedness condition in [22]) and strives to achieve the "right" amount of polyvariance, producing sufficiently many (but not too much) specialised versions for each predicate definition in the original program.

Gallagher in [8] writes that providing adequate global control seems much harder than handling the local level. So, in this paper, it is to the latter, global, level that we (again) turn our attention. In recent work, both authors of the present paper have already, separately, investigated issues in global control of partial deduction. [26] and its extended and slightly revised version [25] focus on termination and provide a quite general, refined framework for global control, different instances of which can be taken as the core of practical systems. [17] on the other hand starts from the central role of characteristic trees in preserving specialisation and determining polyvariance (as first proposed in [10]). It shows how partial deduction can be governed through the use of characteristic atoms, combining the usual atoms to be partially deduced with the characteristic trees to be enforced upon them. Global termination, however, is guaranteed at the cost of imposing an arbitrary, ad hoc depth bound on characteristic trees (as in all earlier partial deduction based on characteristic trees, see e.g. $[10,7,18])$.

In the present paper, we endeavour to obtain the best of both worlds, blending (a slightly adapted version of) the general framework in $[26,25]$ with the use of characteristic atoms and trees as in [17]. We thus obtain a very elegant, sophisticated and precise apparatus for on-line global control of partial deduction.

Below, we first recapitulate some necessary background material and provide motivating examples for our approach in Sect. 2. Subsequently, Sect. 3 contains the formal elaboration of our method, including a partial deduction algorithm, parameterised by its local control, the particular choice of which is left open in this paper. Next, in Sect. 4, we return to the examples in Sect. 2, showing how indeed the method developed in Sect. 3 deals properly with them and leads to greatly improved practical specialisation results. Finally, a possible drawback of the presented approach as well as some connections with related work in supercompilation are briefly discussed. We conclude the paper in Sect. 5 .

In order to remain within reasonable space limits, we have not included any proofs. These can be found in [20], which also contains a section on postprocessing the output of Algorithm 2 as well as a more extensive discussion of experimental results, connections to other work, and assorted topics. 


\section{Ecological Partial Deduction and the Depth Bound Problem}

In what follows, we assume the reader to be familiar with the basic concepts of logic programming and partial deduction, as they are presented in e.g. [21, 22] . Throughout, unless stated explicitly otherwise, the terms "(logic) program" and "goal" will refer to a normal logic program and goal, respectively.

Given a program $P$ and a goal $G$, partial deduction produces a new program $P^{\prime}$ which is $P$ "specialised" to the goal $G$. The underlying technique is to construct "incomplete" SLDNF-trees for a set of atoms $\mathcal{A}$ to be specialised and extract the program $P^{\prime}$ from these incomplete search trees by taking resultants (for each atom in $\mathcal{A}$ a different specialised predicate definition will thus be generated). An incomplete SLDNF-tree is an SLDNF-tree which, in addition to success and failure leaves, may also contain leaves where no literal has been selected for a further derivation step. Leaves of the latter kind will be called dangling. Under the conditions stated in [22], namely closedness and independence, correctness of the specialised program is guaranteed.

In the context of partial deduction, incomplete SLDNF-trees are obtained by applying an unfolding rule, defined as follows:

Definition 1. An unfolding rule $U$ is a function which given a program $P$ and a goal $G$ returns a finite (possibly incomplete) SLDNF-tree ${ }^{1}$ for $P \cup\{G\}$.

\subsection{Ecological Partial Deduction}

The problem of controlling polyvariance in partial deduction boils down to finding a terminating procedure to produce a finite set of atoms $\mathcal{A}$ which satisfies the correctness conditions of [22] while at the same time being as precise as possible (usually the more fine-grained and instantiated the set $\mathcal{A}$ is, the better the potential for specialisation is). Most approaches in the literature so far are based on the syntactic structure of the atoms to be specialised, but it can be shown (see e.g. $[18,17]$ ) that this provides insufficient detail.

$[10,7]$ therefore introduced the notion of a characteristic tree, capturing how atoms are specialised and as such constituting a more refined basis for polyvariance. The following definitions are adapted from $[7,17,18]$.

Definition 2. Let $G_{1}$ be a goal and let $P$ be a program the clauses of which are numbered. Let $G_{1}, \ldots, G_{n}$ be a finite, incomplete $S L D N F$-derivation of $P \cup\left\{G_{1}\right\}$. The characteristic path of the derivation is the sequence $\left(l_{1}, c_{1}\right), \ldots,\left(l_{n-1}, c_{n-1}\right)$, where $l_{i}$ is the position of the selected literal in $G_{i}$, and $c_{i}$ is defined as:

- if the selected literal is an atom, then $c_{i}$ is the number of the clause chosen to resolve with $G_{i}$.

- if the selected literal is $\neg p(\bar{t})$, then $c_{i}$ is the predicate $p$.

The set of all characteristic paths for a given goal $G$ and program $P$ will be denoted by chpaths $(G, P)$.

\footnotetext{
${ }^{1}$ We even allow a trivial SLDNF-tree, i.e. one whose root is a dangling leaf.
} 
Definition 3. Let $G$ be a goal, $P$ a program and $U$ an unfolding rule. Then the characteristic tree $\tau$ of $G$ (in $P$ ) via $U$ is the set of characteristic paths of the non-failing derivations of the incomplete $S L D N F$-tree obtained by applying $U$ to $G$ (in $P$ ). We introduce the notation chtree $(G, P, U)=\tau$. We also say that $\tau$ is a characteristic tree of $G$ (in $P$ ) if it is the characteristic tree for some unfolding rule $U$. Also $\tau$ is a characteristic tree if it is a characteristic tree of some $G$ in some $P$.

Example 1. Let $P$ be the following program:

(1) $\quad$ member $(X,[X \mid T]) \leftarrow$

(2) $\operatorname{member}(X,[Y \mid T]) \leftarrow$ member $(X, T)$

Let $A_{1}=$ member $(a,[a, b])$ and $A_{2}=$ member $\left.(a,[a])\right\}$. Suppose that $A_{1}, A_{2}$ are unfolded as depicted in Fig. 1 . Then both these atoms have the same characteristic tree $\tau=\{((1,1))\}$.

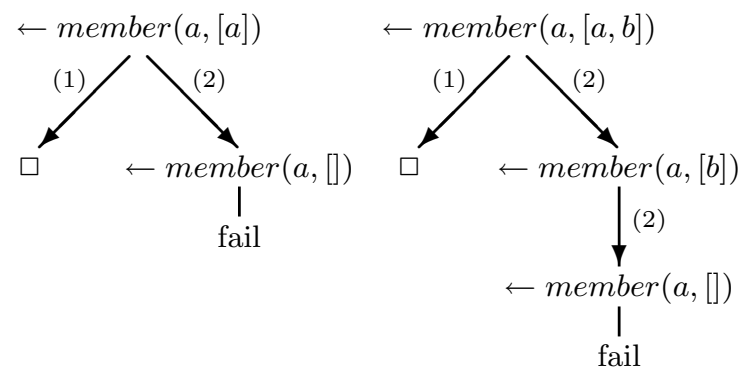

Fig. 1. Incomplete SLDNF-trees for example 1

Note that the characteristic path of an empty derivation is the empty path (), and the characteristic tree of a trivial SLDNF-tree is $\{()\}$. Characteristic trees are an interesting abstraction of SLDNF-trees because they capture the specialisation performed locally by partial deduction. If two atomic goals have the same characteristic tree then the same branches have been pruned by partial deduction and the atoms have been unfolded in the same way (i.e. to the same depth and the same clauses have been resolved with literals in the same position). Furthermore the goals in the bodies of the resultants have the same structure, varying only by the actual call patterns, meaning that in principle a single predicate definition can be used. As such, a characteristic tree is an almost perfect characterisation of the partial deduction of an atom and of the specialisation that has been performed. ${ }^{2}$ For more details about the interest and relevance of characteristic trees, we refer to $[10,7,18,17]$.

\footnotetext{
${ }^{2}$ Sometimes atoms with different characteristic trees have (almost) identical resultants
} (due to independence of the computation rule) and could therefore also be replaced 
When using characteristic trees to control polyvariance, the atoms to be specialised are classified according to their characteristic tree. The basic idea is to have only one specialised version for each characteristic tree. If several atoms have the same characteristic tree, then they are abstracted by a single more general atom. Earlier approaches to partial deduction using characteristic trees [7, 10] have been limited by not being able to preserve characteristic trees in that abstraction process (implying that the generalisation has a different local specialisation behaviour). As shown in $[17,18]$, this can lead to important precision losses as well as non-termination.

The problem has been solved in [17] by simply imposing characteristic trees on the generalised atoms. ${ }^{3}$ This amounts to associating characteristic trees with the atoms to be specialised, thus allowing the preservation of characteristic trees without having to construct intricate generalisations. The rest of this subsection recapitulates and adapts the necessary material from [17].

From now on, throughout the rest of this paper, we implicitly assume the existence of some given finite underlying language $\mathcal{L}$ in which atoms and terms, goals and programs are expressed.

We first introduce the crucial notion of a characteristic atom.

Definition 4. A P-characteristic atom, for a given program $P$, is a couple $(A, \tau)$ consisting of an atom $A$ and a characteristic tree $\tau$ with $\tau \subseteq$ chpaths $(\leftarrow A, P)$.

Often, when the context allows it, we will drop the $P$ annotation and simply refer to characteristic atoms. Also note that $\tau$ is not necessarily a characteristic tree of $\leftarrow A$ in $P$.

Example 2. Let $C A_{1}=(\operatorname{member}(a,[a, b]), \tau), C A_{2}=(\operatorname{member}(a,[a]), \tau)$ with $\tau=\{((1,1))\}$, and let $P$ be the program from Example 1. $C A_{1}$ and $C A_{2}$ are both $P$-characteristic atoms with the same characteristic tree component. The method of [17] will abstract $C A_{1}, C A_{2}$ by $C A_{3}=(\operatorname{member}(a,[a \mid T]),\{((1,1))\})$, i.e. it imposes the characteristic tree $\{((1,1))\}$ on the $m s g$ of the atom components. $C A_{3}$ is also a $P$-characteristic atom, but this time $\{((1,1))\}$ is not a characteristic tree of member $(a,[a \mid T])$ (depending on the unfolding rule the characteristic tree of member $(a,[a \mid T])$ is: $\{((1,1)),((1,2))\}$ or $\{((1,1)),((1,2),(1,1))$, $((1,2),(1,2))\}$ or something even deeper $)$.

The following definition associates a set of concretisations with each characteristic atom.

by a single predicate definition. Normalising characteristic trees (after unfolding) by imposing e.g. a left-to-right ordering of selected literals and delaying the selection of negative literals to the end solves this problem (see also [18]). Thanks to Maurice Bruynooghe for pointing this out. A similar effect can also be obtained by using the trace terms of [?].

${ }^{3}$ Another solution is presented in [18], which is however limited to definite programs and certain unfolding rules, but enjoys a better overall precision. The core ideas of the present paper can also be used to enhance the [18] method in order to eliminate a similar depth bound problem. 
Definition 5. An atom $A$ is a precise concretisation of a P-characteristic atom $\left(A^{\prime}, \tau^{\prime}\right)$ iff $A$ is an instance of $A^{\prime}$ and for some unfolding rule $U$ we have that chtree $(\leftarrow A, P, U)=\tau^{\prime}$. An atom $B$ is a concretisation of $\left(A^{\prime}, \tau^{\prime}\right)$ iff it is an instance of a precise concretisation of $\left(A^{\prime}, \tau^{\prime}\right)$.

A $P$-characteristic atom can thus be seen as standing for a (possibly infinite) set of atoms, namely the concretisations according to the above definition.

Example 3. Take the $P$-characteristic atom $C A_{3}=(\operatorname{member}(a,[a \mid T]),\{((1,1))\})$ from Example 2 . The atoms member $(a,[a])$ and member $(a,[a, b])$ are precise concretisations of $C A_{3}$ (given the unfolding rule of Fig. 1). Also, neither member $(a,[a \mid T])$ nor member $(a,[a, a])$ are concretisations of $C A_{3}$.

A characteristic atom $(A, \tau)$ also uniquely determines a set of resultants:

Definition 6. Let $(A, \tau)$ be a P-characteristic atom. If $\tau \neq\{()\}$ then $\delta(P,(A, \tau))$ is the set of all (necessarily non-failing) SLDNF-derivations for $P \cup\{\leftarrow A\}$ such that their characteristic paths are in $\tau$. If $\tau=\{()\}$ then $\delta(P,(A, \tau))$ is the set of all non-failing $S L D$-derivations for $P \cup\{\leftarrow A\}$ of length $1{ }^{4}$

Definition 7. Let $(A, \tau)$ be a P-characteristic atom. Let $\left\{\delta_{1}, \ldots, \delta_{n}\right\}$ be the $S L D N F$-derivations in $\delta(P,(A, \tau))$ and let $\leftarrow G_{1}, \ldots, \leftarrow G_{n}$ be the goals in the leaves of these derivations. Let $\theta_{1}, \ldots, \theta_{n}$ be the computed answers of the derivations from $\leftarrow A$ to $\leftarrow G_{1}, \ldots, \leftarrow G_{n}$ respectively. Then the set of resultants $\left\{A \theta_{1} \leftarrow G_{1}, \ldots, A \theta_{n} \leftarrow G_{n}\right\}$ is called the partial deduction of $(A, \tau)$ in $P$. Every atom occurring in some of the $G_{i}$ will be called a body atom (in $P$ ) of $(A, \tau)$. We will denote the set of such body atoms by $B A_{P}(A, \tau)$.

For example the partial deduction of (member $(a,[a \mid T]),\{((1,1))\})$ in the program $P$ of Example 1 will be $\{$ member $(a,[a \mid T]) \leftarrow\}$. Note that it is different from any set of resultants that can be obtained for incomplete SLDNF-trees of the ordinary atom member $(a,[a \mid T])$. However the partial deduction is valid for any concretisation (as defined in Def. 5) of (member $(a,[a \mid T]),\{((1,1))\}$ ).

The approach in [17] generates a partial deduction not for a set of atoms but for a set of characteristic atoms. Algorithm 2 below (also) computes exactly such a set of characteristic atoms. The actual code of the specialised program is then produced by unfolding the atoms according to their associated characteristic trees, as described in Definitions 6 and 7.

Of course, the same atom $A$ might occur in several characteristic atoms with entirely different characteristic trees. In order to guarantee correctness of the specialised program, renaming (as well as filtering, see also [9]) is added in [17]. Then, given the following coveredness condition, correctness of the specialised program is established in [17].

Definition 8. Let $P$ be a program and $\mathcal{A}$ a set of characteristic atoms. Then $\mathcal{A}$ is called $P$-covered iff for every characteristic atom in $\mathcal{A}$ each of its body atoms in $P$ is a concretisation of a characteristic atom in $\mathcal{A}$.

\footnotetext{
${ }^{4}$ The reason behind the special treatment of the case $\tau=\{()\}$ is that at least one unfolding step is needed to avoid the problematic resultant $A \leftarrow A$ in Definition 7.
} 


\subsection{The Depth Bound Problem}

When, for the given program, query and unfolding rule, the above sketched method generates a finite number of different characteristic trees, its global control regime guarantees termination and correctness of the specialised program as well as "perfect" 5 polyvariance: For every predicate, exactly one specialised version is produced for each of its different associated ${ }^{6}$ characteristic trees. Now, [17], as well as all earlier approaches based on characteristic trees $([10,7,18])$, achieves the mentioned finiteness condition at the cost of imposing an ad hoc (typically very large) depth bound on characteristic trees. However, for a fairly large class of realistic programs (and unfolding rules), the number of different characteristic trees generated, is not naturally bounded. In those cases, the underlying depth bound will have to ensure termination, meanwhile propagating its ugly, ad hoc nature into the resulting specialised program. We illustrate this problem through some examples, setting out with a slightly artificial, but very simple one.

Example 4. The following is the well known reverse with accumulating parameter where a list type check on the accumulator has been added.

$$
\begin{aligned}
& \text { (1) } \operatorname{rev}([], A c c, A c c) \leftarrow \\
& \text { (2) } \operatorname{rev}([H \mid T], A c c, \operatorname{Res}) \leftarrow l s(A c c), \operatorname{rev}(T,[H \mid A c c], \operatorname{Res}) \\
& \text { (3) } \operatorname{ls}([]) \leftarrow \\
& \text { (4) } l s([H \mid T]) \leftarrow l s(T)
\end{aligned}
$$

As can be noticed in Fig. 2, (determinate $([10,7,18])$ and well-founded $([3,24$, $23]$ ), among others) unfolding produces an infinite number of different characteristic atoms, all with a different characteristic tree. Imposing a depth bound of say 100 , we obtain termination, but the algorithm produces 100 different reverse versions and the specialised program looks like:

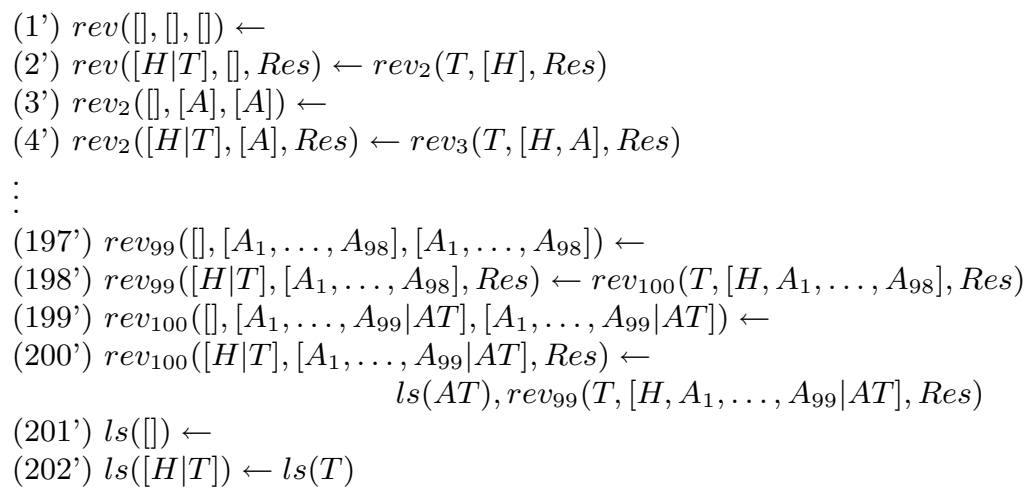

This program is certainly far from optimal and clearly exhibits the ad hoc nature of the depth bound.

\footnotetext{
${ }^{5}$ W.r.t. local precision, see $[18,17]$.

${ }^{6}$ I.e. a characteristic tree associated to an atom with this predicate symbol.
} 


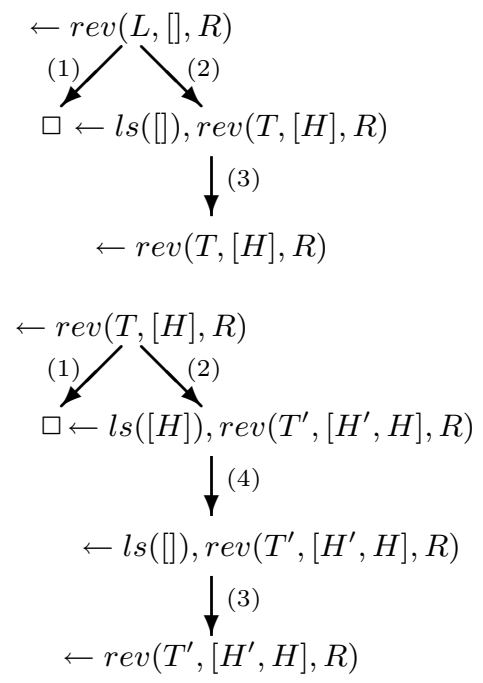

In general:
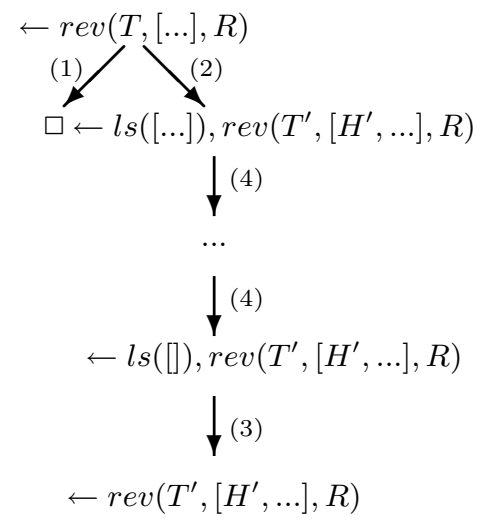

Fig. 2. SLD-trees for Example 4

Situations like the above typically arise when an accumulating parameter influences the computation, because then the growing of the accumulator causes a corresponding growing of the characteristic trees. To be fair, it must be admitted that with most simple programs, this is not the case. For instance, in the standard reverse with accumulating parameter, the accumulator is only copied in the end, but never influences the computation. As illustrated by Example 4 above, this state of affairs will often already be changed when one adds type checking in the style of [11] to even the simplest logic programs.

Among larger and more sophisticated programs, cases like the above become more and more frequent, even in the absence of type checking. ${ }^{7}$ For instance, in an explicit unification algorithm, one accumulating parameter is the substitution built so far. It heavily influences the computation because new bindings have to be added and checked for compatibility with the current substitution. Another example is the "mixed" meta-interpreter of $[12,19]$ (sometimes called InstanceDemo; part of it is depicted in Fig. 3) for the ground representation in which the goals are "lifted" to the non-ground representation for resolution. To perform the lifting, an accumulating parameter is used to keep track of the variables that have already been encountered. This accumulator influences the computation: Upon encountering a new variable, the program inspects the accumulator.

\footnotetext{
${ }^{7}$ Especially since efficiently written programs often use accumulating parameters.
} 


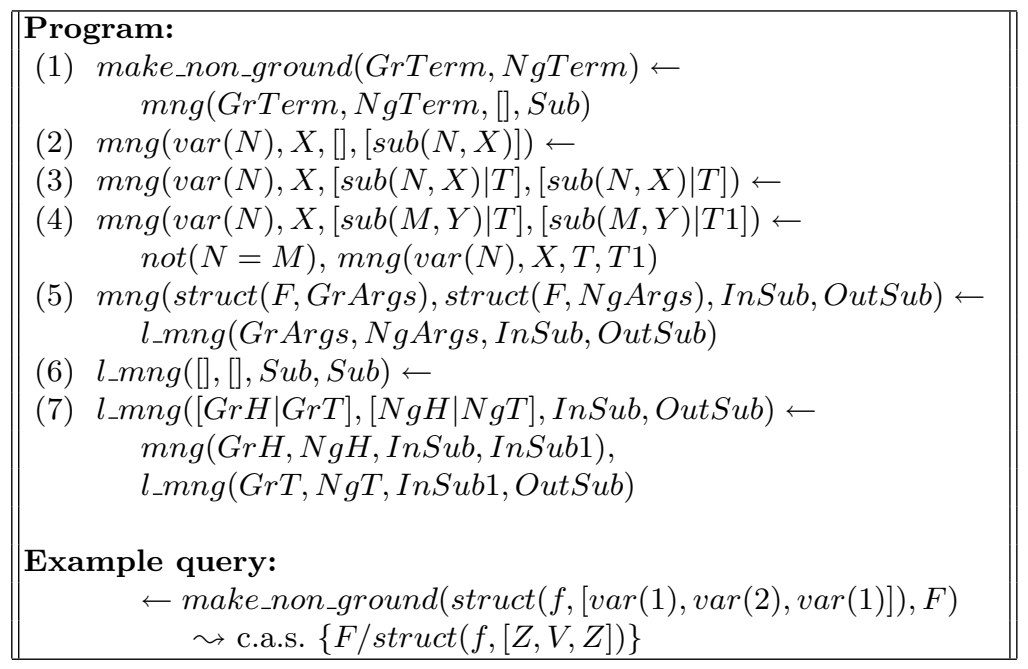

Fig. 3. Lifting the ground representation

Example 5. Let $A=l_{-} m n g(L g, L n,[\operatorname{sub}(N, X)], S)$ and $P$ be the program of Fig. 3 (this situation arose in a real-life experiment). As can be seen in Fig. 4, unfolding $A$ (e.g. using well-founded measures) causes the addition of the atom l_mng $(T g, T n,[\operatorname{sub}(N, X), \operatorname{sub}(J, H n)], S)$ at the global (control) level. Notice that the third argument has grown (i.e. we have an accumulator).

So, when in turn unfolding $l \_m n g(T g, T n,[\operatorname{sub}(N, X), \operatorname{sub}(J, H n)], S)$, we will obtain a deeper characteristic tree (because $m n g$ traverses the third argument and thus needs one more step to reach the end) which will have as one of its leaves the atom $l \_m n g\left(T g^{\prime}, T n^{\prime},\left[\operatorname{sub}(N, X), \operatorname{sub}(J, H n), \operatorname{sub}\left(J^{\prime}, H n^{\prime}\right)\right], S\right)$. An infinite sequence of ever growing characteristic trees results and again, as in Example 4, we obtain non-termination without a depth bound, and very unsatisfactory (ad hoc) specialisations with it.

Summarising, computations influenced by one or more growing data structures are by no means rare and will, very often, lead to ad hoc behaviour of partial deduction where the global control is founded on characteristic trees with a depth bound. In the next section, we show how this annoying depth bound can be lifted without endangering termination.

\section{Partial Deduction using Global Trees}

\subsection{Introduction}

A general framework for global control, not relying on any depth bounds, is proposed in $[26,25]$. Marked trees (m-trees) are introduced to register descendency relationships among atoms at the global level. The overall tree is then kept finite 

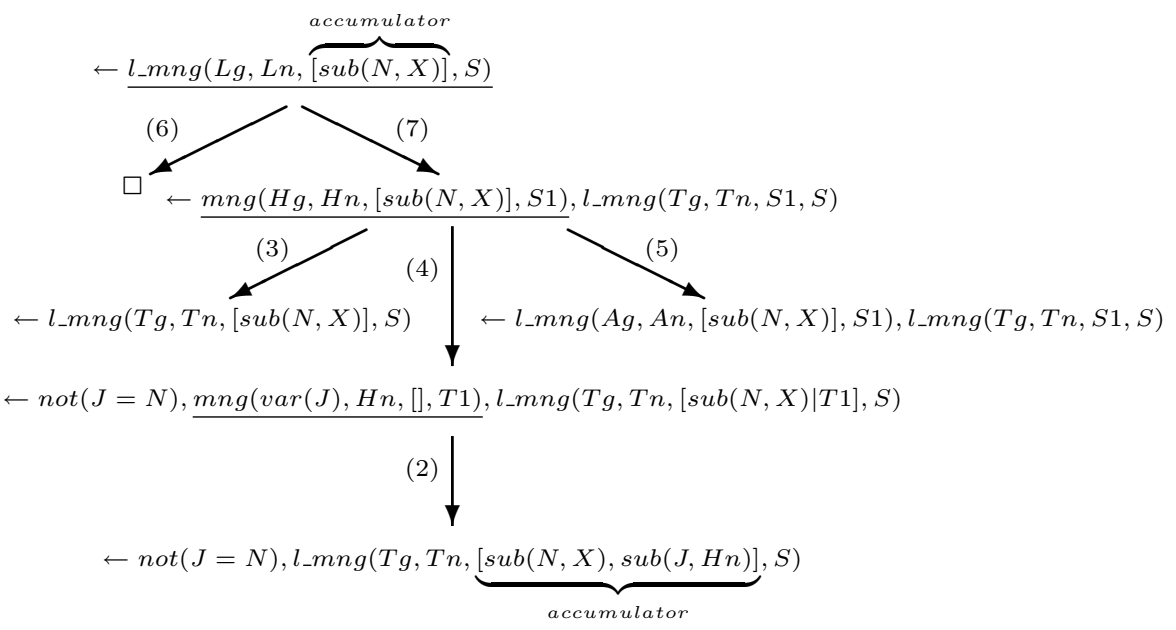

Fig. 4. Accumulator growth in Example 5

through ensuring monotonicity of well-founded measure functions and termination of the algorithm follows, provided the generalisation operation (on atoms) is similarly well-founded. It is to this framework that we now turn for inspiration on how to solve the depth bound problem uncovered in Subsect. 2.2.

The basic idea will be to watch over the evolution of characteristic trees associated to atoms along the branches of the global tree. Obviously, just measuring the depth of characteristic trees would be far too crude: Global branches would be cut off prematurely and entirely unrelated atoms could be mopped together through generalisation, resulting in completely unacceptable specialisation losses. No, as can be seen in Fig. 2, we need a more refined measure which would somehow spot when a characteristic tree (piecemeal) "contains" characteristic trees appearing earlier in the same branch of the global tree. If such a situation arises (as it indeed does in Example 4), it seems reasonable to stop expanding the global tree, generalise the offending atoms and produce a specialised procedure for the generalisation instead.

However, a closer look at the following variation of Example 5 shows that also this approach would sometimes overgeneralise and consequently fall short of providing sufficiently detailed polyvariance.

Example 6. Reconsider the program in Fig. 3, and suppose that local control uses determinate unfolding. Let us now start partial deduction for the atom $A=\operatorname{mng}(G, \operatorname{struct}(c l,[\operatorname{struct}(f,[X, Y]) \mid B]),[], S)$ (also this situation arose in a real-life experiment). When unfolding $A$ (see Fig. 5), we obtain an SLDNFtree containing the atom $m n g(H, \operatorname{struct}(f,[X, Y]),[], S 1)$ in one of its leaves. If we subsequently (determinately) unfold the latter atom, we obtain a tree that is "larger" than its predecessor, also in the more refined sense. Potential nontermination would therefore be detected and a generalisation operation executed. However, the atoms in the leaves of the second tree are more general than those 
already met, and simply continuing partial deduction without generalisation will lead to natural termination without any depth bound intervention.

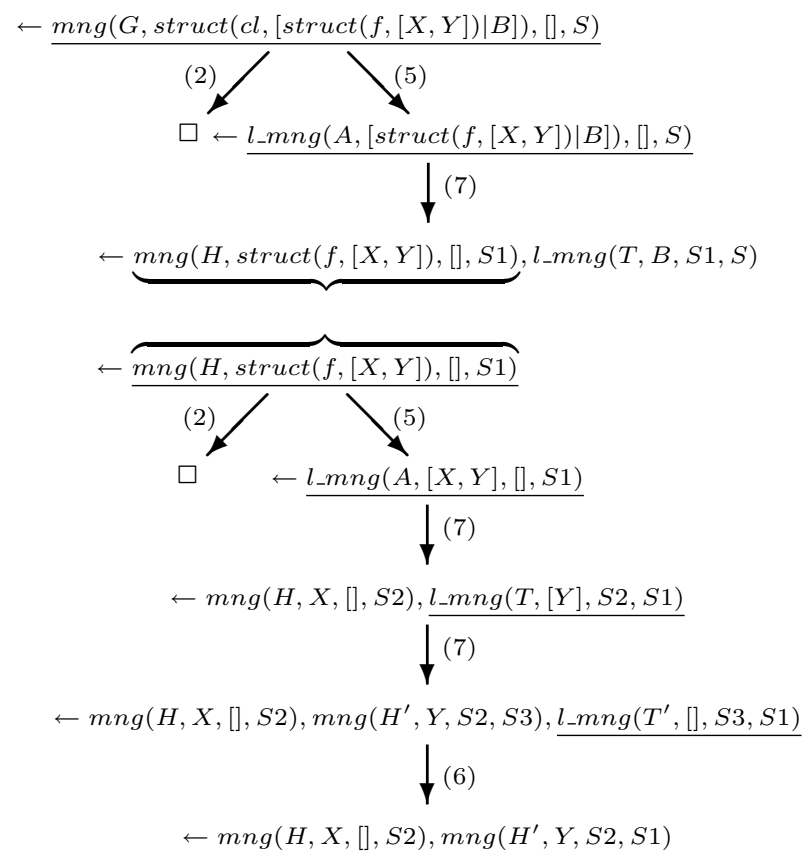

Fig. 5. SLD-trees for Example 6

Example 6 demonstrates that only measuring growth of characteristic trees, even in a refined way, does not always lead to satisfactory specialisation. ${ }^{8}$ Luckily, the same example also suggests a solution to this problem: Rather than measuring and comparing characteristic trees, we will scrutinise entire characteristic atoms, comparing both the syntactic content of the ordinary atoms they contain and the associated characteristic trees. Accordingly, the global tree nodes will not be labeled by plain atoms as in $[26,25]$, but by entire characteristic atoms.

The rest of this section, then, contains the formal elaboration of this new approach.

${ }^{8}$ In fact, whenever the (local) unfolding rule does not unfold "as deeply as possible" (for whatever reason), then a growing characteristic tree might simply be caused by splitting the "maximally deep tree" in such a way that the second part "contains" the first part. 


\subsection{More on Characteristic Atoms}

Generalising Characteristic Atoms. We extend the notions of variants, instances and generalisations, familiar for ordinary atoms, ${ }^{9}$ to characteristic trees and atoms:

Definition 9. A characteristic tree $\tau_{1}$ is more general than another characteristic tree $\tau_{2}$, denoted by $\tau_{1} \preceq \tau_{2}$, iff $\tau_{2}$ can be obtained by attaching subtrees to the leaves of $\tau_{1}$. A characteristic atom $\left(A_{1}, \tau_{1}\right)$ is more general than another characteristic atom $\left(A_{2}, \tau_{2}\right)$, denoted by $\left(A_{1}, \tau_{1}\right) \preceq\left(A_{2}, \tau_{2}\right)$, iff $A_{1} \preceq A_{2}$ and $\tau_{1} \preceq \tau_{2}$. Also $\left(A_{1}, \tau_{1}\right)$ is a variant of $\left(A_{2}, \tau_{2}\right)$, denoted by $\left(A_{1}, \tau_{1}\right) \equiv\left(A_{2}, \tau_{2}\right)$, iff $\left(A_{1}, \tau_{1}\right) \preceq\left(A_{2}, \tau_{2}\right)$ and $\left(A_{2}, \tau_{2}\right) \preceq\left(A_{1}, \tau_{1}\right)$. Finally, $C A_{1} \prec C A_{2}$ holds when $C A_{1} \preceq C A_{2}$ but not $C A_{1} \equiv C A_{2}$.

Note that $\{()\}$, a characteristic tree containing a single, empty path, can be extended into a more specific characteristic tree, while the empty characteristic tree $\emptyset=\{\}$ cannot.

Example \%. Let $\tau_{1}=\{((1,3))\}, \tau_{2}=\{((1,3),(2,4))\}$ and $\tau_{3}=\{((1,3)),((1,4))\}$, then we have that $\tau_{1} \preceq \tau_{2}$ and $\tau_{1} \prec \tau_{2}$ but not that $\tau_{1} \preceq \tau_{3}$ nor $\tau_{2} \preceq \tau_{3}$.

The following proposition shows that the above definition makes sense wrt the set of ordinary atoms represented by characteristic atoms.

Proposition 1. Let $C A_{1}, C A_{2}$ be two characteristic atoms such that $C A_{1} \preceq$ $C A_{2}$. Then every atom $A$ which is a concretisation of $C A_{2}$ is also a concretisation of $C A_{1}$.

Finally, we extend the notion of most specific generalisation (msg) to characteristic trees and atoms:

Definition 10. Let $\tau_{1}, \tau_{2}$ be two P-characteristic trees. Then $m s g\left(\tau_{1}, \tau_{2}\right)$ is the most specific characteristic tree which is more general than both $\tau_{1}$ and $\tau_{2}$.

Let $\left(A_{1}, \tau_{1}\right),\left(A_{2}, \tau_{2}\right)$ be two characteristic atoms. Then $m s g\left(\left(A_{1}, \tau_{1}\right),\left(A_{2}, \tau_{2}\right)\right)=$ ( $\left.m s g\left(A_{1}, A_{2}\right), m s g\left(\tau_{1}, \tau_{2}\right)\right)$.

Note that the above $m s g$ for characteristic atoms is still unique up to variable renaming. Its further extension to sets of characteristic atoms (rather than just pairs) is straightforward, and will not be included explicitly.

Example 8. Given $\tau_{1}=\{((1,3))\}, \tau_{2}=\{((1,3),(2,4))\}, \tau_{3}=\{((1,3)),((1,4))\}$, $\tau_{4}=\{((1,3),(2,4)),((1,3),(2,5))\}$, we have that $m s g\left(\tau_{1}, \tau_{2}\right)=\tau_{1}, m s g\left(\tau_{1}, \tau_{3}\right)=$ $m s g\left(\tau_{2}, \tau_{3}\right)=\{()\}$ and $m s g\left(\tau_{2}, \tau_{4}\right)=\tau_{1}$.

Ordering Characteristic Atoms. We now proceed to introduce an order relation on characteristic atoms. It will be instrumental in guaranteeing termination of the partial deduction method to be presented.

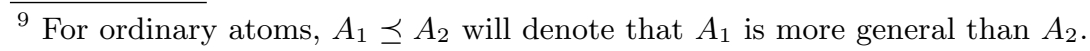


Definition 11. A set $V, \leq_{V}$ is called well-quasi-ordered (wqo) iff for any infinite sequence of elements $e_{1}, e_{2}, \ldots$ in $V$ there are $i<j$ such that $e_{i} \leq_{V} e_{j}$. We also say that $\leq_{V}$ is a well-quasi order (wqo) on $V$.

An interesting wqo is the homeomorphic embedding relation $\unlhd$ of [30] (where it is adapted from [6]).

Definition 12. The homeomorphic embedding relation $\unlhd$ on atoms and terms ${ }^{10}$ is defined inductively as follows:

1. $X \unlhd Y$ for all variables $X, Y$

2. $s \unlhd f\left(t_{1}, \ldots, t_{n}\right)$ if $s \unlhd t_{i}$ for some $i$

3. $f\left(s_{1}, \ldots, s_{n}\right) \unlhd f\left(t_{1}, \ldots, t_{n}\right)$ if $\forall i \in\{1, \ldots, n\}: s_{i} \unlhd t_{i}$.

Example 9. We have: $p(a) \unlhd p(f(a)), X \unlhd X, p(X) \unlhd p(f(Y)), p(X, X) \unlhd p(X, Y)$ and $p(X, Y) \unlhd p(X, X)$.

Proposition 2. The relation $\unlhd$ is a wqo on the set of atoms and terms.

The intuition behind Definition 12 is that when some structure re-appears within a larger one, it is homeomorphically embedded by the latter. As is argued in [30], this provides a good starting point for detecting growing structures created by (hence) possibly non-terminating processes.

However, as can be observed in Example 9, the homeomorphic embedding relation $\unlhd$ as defined in Definition 12 is rather crude wrt variables. In fact, all variables are treated as if they were the same variable, a practice which is clearly undesirable in a logic programming context. Intuitively, in the above example, $p(X, Y) \unlhd p(X, X)$ is acceptable, while $p(X, X) \unlhd p(X, Y)$ is not. $^{11}$

To remedy the problem, we refine the above introduced homeomorphic embedding as follows:

Definition 13. Let $A, B$ be atoms or terms. Then $B$ (strictly homeomorphically) embeds $A$, written as $A \unlhd^{*} B$, iff $A \unlhd B$ and $A$ is not a strict instance of $B$.

Example 10. We now have that $p(X, Y) \unlhd^{*} p(X, X)$ but not $p(X, X) \unlhd^{*} p(X, Y)$. Note that still $X \unlhd^{*} Y$ and $X \unlhd^{*} X$.

Proposition 3. The relation $\unlhd^{*}$ is a wqo on the set of atoms and terms.

We now extend the embedding relation of Definition 13 to characteristic atoms. One way to obtain a wqo is to first define a term representation of characteristic trees and then use the embedding relation $\unlhd^{*}$ with this term representation.

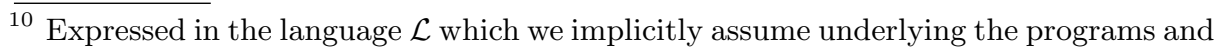
queries under consideration. Remember that it contains only finitely many constant, function and predicate symbols! This property is of crucial importance for the proofs in $[20]$.

${ }^{11} p(X, X)$ can be seen as standing for something like and $(e q(X, Y), p(X, Y))$ which clearly embeds $p(X, Y)$, but the reverse does not hold. 
Definition 14. By $\lceil$.$\rceil we denote an injective, monotonic { }^{12}$ mapping from characteristic trees to (ordinary) atoms.

Such a mapping can be easily constructed by representing leaves of the tree by variables. For example we could have $\lceil\{((1,3))\}\rceil=\operatorname{select}(1,[\operatorname{match}(3, X)])$ and $\lceil\{((1,3),(2,4))\}\rceil=\operatorname{select}(1,[\operatorname{match}(3, \operatorname{select}(2,[\operatorname{match}(4, X)]))]) .{ }^{13}$

Definition 15. Let $\left(A_{1}, \tau_{1}\right)$ and $\left(A_{2}, \tau_{2}\right)$ be two characteristic atoms. We say that $\left(A_{2}, \tau_{2}\right)$ embeds $\left(A_{1}, \tau_{1}\right)$, denoted by $\left(A_{1}, \tau_{1}\right) \unlhd^{*}\left(A_{2}, \tau_{2}\right)$, iff $A_{1} \unlhd^{*} A_{2}$ and $\left\lceil\tau_{1}\right\rceil \unlhd^{*}\left\lceil\tau_{2}\right\rceil$.

Proposition 4. Let $\mathcal{A}$ be a set of P-characteristic atoms. Then $\mathcal{A}, \unlhd^{*}$ is wellquasi-ordered.

Finally, we consider the relationship between $\unlhd^{*}$ and $\preceq$ on characteristic atoms.

Proposition 5. Let $C A_{1}, C A_{2}, C A_{3}$ be characteristic atoms such that $C A_{3} \preceq$ $C A_{2}$. Then $C A_{1} \unlhd^{*} C A_{3} \Rightarrow C A_{1} \unlhd^{*} C A_{2}$.

So, a generalisation of a given characteristic atom will only embed characteristic atoms already embedded by the given one.

Proposition 6. Let $C A_{1}, C A_{2}$ be characteristic atoms such that $C A_{2} \preceq C A_{1}$. Then $C A_{1} \unlhd^{*} C A_{2}$ iff $C A_{1} \equiv C A_{2}$.

Proposition 7. Let $C A_{1}, \ldots, C A_{n}$ be characteristic atoms and assume that $M=m s g\left(C A_{1}, \ldots, C A_{n}\right) .{ }^{14}$ Then the following three statements are equivalent (where $1 \leq i \leq n$ ):

$$
\begin{aligned}
& \text { 1. } C A_{i} \unlhd^{*} M \\
& \text { 2. } C A_{i} \equiv M \\
& \text { 3. } C A_{i} \preceq C A_{1}, \ldots, C A_{i} \preceq C A_{n}
\end{aligned}
$$

Properties 5 and 7 will be used to prove termination of the partial deduction algorithm in Sect. 3.4. Note that Propositions 6 and 7 do not hold for the $\unlhd$ relation (which makes $\unlhd$ less suitable for ensuring termination of partial deduction).

\subsection{Global Trees}

In this subsection, we adapt and instantiate the m-tree concept presented in [26, $25]$ according to our particular needs in this paper.

Definition 16. A global tree $\gamma_{P}$ for a program $P$ is a (finitely branching) tree where nodes can be either marked or unmarked and each node carries a label which is a P-characteristic atom.

${ }^{12}$ I.e. if $\tau_{1} \prec \tau_{2}$ then $\left\lceil\tau_{1}\right\rceil \prec\left\lceil\tau_{2}\right\rceil$.

${ }^{13}$ Note that $\{((1,3))\} \prec\{((1,3),(2,4))\}$ and indeed $\lceil\{((1,3))\}\rceil \prec\lceil\{((1,3),(2,4))\}\rceil$.

${ }^{14}$ As we in fact already did in the pair-wise case, we slightly abuse notation by not writing $m s g\left(\left\{C A_{1}, \ldots, C A_{n}\right\}\right)$, while that is of course actually what is intended. 
In other words, a node in a global tree $\gamma_{P}$ will look as follows: $\left(n, \operatorname{mark},\left(A, \tau_{A}\right)\right)$, where $n$ is the node identifier, mark an indicator that can take the values $u$ or $m$ designating whether the node is marked or not, and the $P$-characteristic atom $\left(A, \tau_{A}\right)$ is the node's label. Informally, a marked node corresponds to a characteristic atom which has already been treated by the partial deduction algorithm. We will often omit the $P$ subscript when it is either clear from or not relevant in the particular context we are considering.

In the sequel, we consider a global tree $\gamma$ partially ordered through the usual ancestor_node $>_{\gamma}$ descendent_node relationship. Given a node $n \in \gamma$, we denote by $A n c_{\gamma}(n)$ the set of its $\gamma$ ancestor nodes (including itself).

Let $\gamma_{P}$ be a global tree. Then we will henceforth denote as $L_{\gamma_{P}}$ the set of its labels. And for a given node $n$ in a tree $\gamma$, we will refer to its label by $l_{n}$.

Definition 17. Let $\gamma$ be a global tree. Then we define its associated label mapping $f_{\gamma}$ as the one-to-one mapping $f_{\gamma}: \gamma,>_{\gamma} \rightarrow L_{\gamma}, \unlhd^{*}$ such that $n \mapsto l_{n} . f_{\gamma}$ will be called non-monotonic iff $\exists n_{1}, n_{2}$ such that $n_{1}>_{\gamma} n_{2}$ and $l_{n_{1}} \unlhd^{*} l_{n_{2}}$.

Definition 18. We call a global tree $\gamma$ well-quasi-ordered if $f_{\gamma}$ is not nonmonotonic.

Theorem 1. A global tree $\gamma$ is finite if it is well-quasi-ordered.

\subsection{An Algorithm for Partial Deduction}

In this subsection, we present the actual partial deduction algorithm where global control is imposed through characteristic atoms in a global tree.

We first introduce the following definition:

Definition 19. Let $A$ be an ordinary atom, $U$ an unfolding rule and $P$ a program. Then chatom $(A, P, U)=(A, \tau)$ where chtree $(\leftarrow A, P, U)=\tau$.

A formal description of the algorithm can be found in Fig. 6. Please note that it is parameterised by an unfolding rule $U$, thus leaving the particulars of local control unspecified. Without loss of generality, we suppose the initial goal to consist of a single atom.

As in e.g. [8, 26, 17], Algorithm 2 does not output a specialised program, but rather a set of (characteristic) atoms from which the actual code can be generated in a straightforward way. Most of the algorithm is self-explanatory, except perhaps the For-loop. In $\mathcal{B}$, all the characteristic atoms are assembled, corresponding to the atoms occurring in the leaves of the SLDNF-tree built (locally, of course) for $A_{L}$ according to $\tau_{A_{L}}$. Elements of $\mathcal{B}$ are subsequently inserted into $\gamma$ as (unmarked) child nodes of $L$ if they do not embed the label of $n$ or any of its ancestor nodes. If one does, and it is an instance of $n$ 's label or that of an ancestor of $n$, then it is simply not added to $\gamma$. Finally, if a characteristic atom $\left(B, \tau_{B}\right) \in \mathcal{B}$ does embed an ancestor label, but there is no more general characteristic atom to be found labelling any of the ancestor nodes, then $n$ receives a child node carrying as label the most specific generalisation of $\left(B, \tau_{B}\right)$ and all 


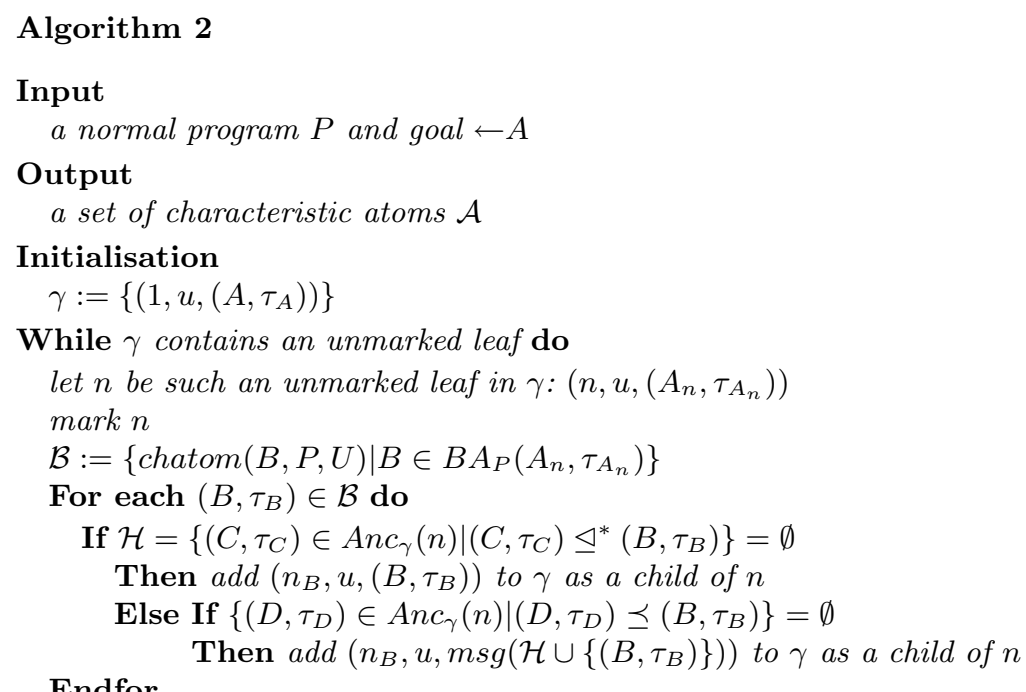

Endfor

Endwhile

$\mathcal{A}:=L_{\gamma}$

Fig. 6. Partial deduction with global trees.

embedded ancestor labels. The latter case is of course the most interesting: Simply adding a node labelled $\left(B, \tau_{B}\right)$ would endanger termination. Adding the $\mathrm{msg}$ label instead secures finiteness, while trying to preserve as much information as seems possible. ${ }^{15}$ We obtain the following theorems:

Theorem 3. Algorithm 2 always terminates.

Theorem 4. Let $P$ be a program, input to Algorithm 2 , and $\mathcal{A}$ the corresponding set of characteristic atoms produced as output. Then $\mathcal{A}$ is $P$-covered.

From Theorem 4, correctness of the specialisation follows as in [17].

Finally, we have developed a post-processing phase reducing the polyvariance to some minimal level without removing any of the specialisation performed by the partial deduction described in Algorithm 2 (see Sect. 3.5 of [20]).

\section{Experimental Results and Discussion}

Creating a fully fledged implementation of Algorithm 2 and the above described post-processing is the subject of ongoing work. However, to preliminarily check whether the developed ideas actually lead to improved practical results, we enhanced the system used for ecological partial deduction in [17] with global

${ }^{15}$ Further enhancing precision through even more cautious generalisation will be a topic of future research. 
control through embedding and generalisation on characteristic atoms. We experimented with the result (denoted by eco-embed), comparing its performance with ecological partial deduction as in [17]. In the latter case, various depth bounds were imposed on the (local) SLDNF-trees in order to ensure termination (hence the notation $e c o-d b_{--}$). Finally, in the experiments described below, local control is always based on the embedding relation on the atoms in the proof tree structure (i.e. it checks whether selected literals in covering ancestors, see [3], are embedded), possibly cut off by the imposed depth bound.

We report on three experiments. In the first one, we specialised the reverse (with type checking) program of Example 4 for the atom $\operatorname{rev}(L,[], R)$. The second and third experiment involved the "lifting" solve meta-interpreter ([12, 19]). The program depicted in Fig. 3 is actually an excerpt from its code. Experiment 2 consisted in specialising this program for solve([" $c 1 ", " c 2 ", U]$, $[\operatorname{struct}(f a,[X, Y])])$, where " $e$ " denotes the ground representation of an expression $e, c 1$ denotes the clause $f a(X, Y) \leftarrow p(X, Y), m(X)$ and $c 2$ the clause $m o(X, Y) \leftarrow p(X, Y), f(X)$. Finally, in Experiment 3, we specialised the same solve for the atom solve ([" c1", "c2"," $p(a, b) \leftarrow ", " m(a) \leftarrow "],[\operatorname{struct}(f a,[X, Y])])$.

The first two experiments illustrate the problems encountered in Examples 4 and 5 , and have to rely on a depth-bound for termination when using ecological partial deduction as in [17]. The third experiment does not require a depth bound, but illustrates another (well-known) adverse effect of using depth bounds.

The results are summarised in Tables 1,2 and 3. The experiments were performed using Prolog by BIM on a Sparc Classic running Solaris. The size of the compiled code is expressed in units, 1 unit corresponding to 4.08 bytes on a Sparc Classic. The (compiled code) run times were obtained by using the time/2 predicate of Prolog by BIM on an extensive number of queries.

Experiment 1 shows that the additional polyvariance produced by using a depth bound to ensure termination does not pay off in efficiency but increases the code size unnecessarily. Experiment 2 illustrates this even more poignantly. The amount of polyvariance produced by the method $e c o-d b 50$ was even so big that we could not complete the partial deduction. ${ }^{16}$

In Experiment 3, it is interesting to note that, even with a depth bound of 50 and for this very simple object program, we do not yet get the optimal result ! So, for Experiment 2, the depth bound of 50 generates way too much polyvariance, while for Experiment 3, the depth bound of 50 is not sufficient to guarantee optimal specialisation of the same program! Concluding, it seems very likely that the global control improvements proposed in this paper will indeed pay off in partial deduction practice.

Note that the current system (eco-embed) does not yet structure the characteristic atoms in a global tree, but still just puts them in a set as in [17]. For the above described experiments, this had no influence, but generalisation with non-ancestors may in general severely limit specialisation potential.

$\overline{16}$ After running for over several hours, producing 134 different predicates and generalising 80 times, eco-db50 overflowed the heap limit of 4.5 Megabytes. 


\begin{tabular}{|c|c|c|r|}
\hline Method & Run Time & \#Clauses/\#Predicates & Compiled Code Size \\
\hline original & $6.7 \mathrm{~s}$ & $4 / 2$ & $203 \mathrm{u}$ \\
eco-embed & $6.7 \mathrm{~s}$ & $6 / 3$ & $331 \mathrm{u}$ \\
eco-db10 & $6.7 \mathrm{~s}$ & $23 / 12$ & $1955 \mathrm{u}$ \\
eco-db50 & $7.1 \mathrm{~s}$ & $103 / 52$ & $29573 \mathrm{u}$ \\
eco-db100 & $7.5 \mathrm{~s}$ & $203 / 102$ & $122391 \mathrm{u}$ \\
\hline
\end{tabular}

Table 1. Experiment 1, rev program

\begin{tabular}{|c|c|c|r|}
\hline Method & Run Time & \#Clauses/\#Predicates & Compiled Code Size \\
\hline original & $3.07 \mathrm{~s}$ & $16 / 8$ & $1328 \mathrm{u}$ \\
eco-embed & $1.42 \mathrm{~s}$ & $138 / 26$ & $26390 \mathrm{u}$ \\
eco-db10 & $1.84 \mathrm{~s}$ & $328 / 68$ & $65863 \mathrm{u}$ \\
eco-db50 & $-\mathrm{s}$ & $-/ \geq 134$ & $-\mathrm{u}$ \\
\hline
\end{tabular}

Table 2. Experiment 2, solve program

\begin{tabular}{|c|c|c|r|}
\hline Method & Run Time & \#Clauses/\#Predicates & Compiled Code Size \\
\hline original & $2.44 \mathrm{~s}$ & $16 / 8$ & $1328 \mathrm{u}$ \\
eco-embed & $0.04 \mathrm{~s}$ & $1 / 1$ & $77 \mathrm{u}$ \\
eco-db10 & $1.63 \mathrm{~s}$ & $34 / 19$ & $4164 \mathrm{u}$ \\
eco-db50 & $0.06 \mathrm{~s}$ & $8 / 4$ & $1062 \mathrm{u}$ \\
\hline
\end{tabular}

Table 3. Experiment 3, solve program for another query

A possible drawback of the global control method as laid out in Sect. 3, might be its considerable complexity. Indeed, first, ensuring termination through a well-quasi-ordering is structurally much more costly than the alternative of using a well-founded ordering. The latter only requires comparison with a single "ancestor" object and can be enforced without any search through "ancestor lists" (see [23]). Testing for well-quasi-ordering, however, unavoidably does entail such searching and repeated comparisons with several ancestors. Moreover, in our particular case, checking $\unlhd^{*}$ on characteristic atoms is in itself a quite costly operation, adding considerably to the innate complexity of maintaining a wellquasi-ordering. It remains therefore to be seen whether a global control such as the one above can be used (or approximated in an efficient way) in circumstances where speed (or complexity) of the transformation is an important factor.

We conclude this section with a brief discussion on the relation between our global control and what may be termed thus in supercompilation $([31,32,30])$. We already pointed out that the inspiration for using $\unlhd$ derives from [30]. In that paper, a generalisation strategy for positive supercompilation (no negative information propagation while driving) is proposed. It uses $\unlhd$ to compare nodes in a marked partial process tree (a notion roughly corresponding to marked or global trees in partial deduction). These nodes, however, only contain syntactical information. It is our current understanding that both the addition of something similar to characteristic trees and the use of the refined $\unlhd^{*}$ embedding can lead to improvements of the method proposed in [30]. Finally, we return to an observation made in [25]: Neighbourhoods of order " $\mathrm{n}$ " in (full) supercompilation ([32]), are essentially the same as classes of atoms (or goals) with an identical 
depth $n$ characteristic tree. Adapting our technique for supercompilation would therefore probably allow to remove the depth bound on neighbourhoods.

\section{Conclusion}

In this paper, we have developed a sophisticated on-line global control technique for partial deduction of normal logic programs. Importing and adapting $\mathrm{m}$-trees from $[26,25]$, we have overcome the need for a depth bound on characteristic trees to guarantee termination of partial deduction as proposed in [17]. Plugging in a depth bound free local control strategy (see e.g. [3,23]), we thus obtain a fully automatic, concrete partial deduction method that always terminates and produces precise and reasonable polyvariance, without resorting to any ad hoc techniques. To the best of our knowledge, this is the very first such method.

Along the way, we have defined generalisation and embedding on characteristic atoms, refining the homeomorphic embedding relation $\unlhd$ from [30] into $\unlhd^{*}$, and showing that the latter is more suitable in a logic programming setting. Initial experiments with a partial implementation of the method showed its great practical value; A fully fledged implementation and further experimentation are the subjects of ongoing work.

\section{Acknowledgements}

We thank the following persons for interesting comments and/or stimulating discussions on this work: Eddy Bevers, Danny De Schreye, André de Waal, Robert Glück, Neil Jones, Jesper Jørgensen, Torben Mogensen, Kristian Nielsen, David Sands and Morten Heine Sørensen. André de Waal, Jesper Jørgensen and an anonymous referee gave useful feedback on a draft version of this paper. Alain Callebaut provided helpful information on benchmarking Prolog by BIM pro-

grams. Finally, we gratefully acknowledge Danny De Schreye's (almost) never failing enthusiasm and support.

\section{References}

1. K. Benkerimi and J. W. Lloyd. A partial evaluation procedure for logic programs. In S. Debray and M. Hermenegildo, editors, Proceedings NACLP'90, pages 343358, Austin, Texas, October 1990. MIT Press.

2. R. Bol. Loop checking in partial deduction. Journal of Logic Programming, 16(1\&2):25-46, 1993.

3. M. Bruynooghe, D. De Schreye, and B. Martens. A general criterion for avoiding infinite unfolding during partial deduction. New Generation Computing, 11(1):47$79,1992$.

4. C. Consel and O. Danvy. Tutorial notes on partial evaluation. In Proceedings POPL'93, Charleston, South Carolina, January 1993. ACM.

5. D. De Schreye, M. Leuschel, and B. Martens. Tutorial on program specialisation (abstract). In J.W. Lloyd, editor, Proceedings ILPS'95, pages 615-616, Portland, Oregon, December 1995. MIT Press. 
6. N. Dershowitz and J.-P. Jouannaud. Rewrite systems. In J. van Leeuwen, editor, Handbook of Theoretical Computer Science, Vol. B, pages 243-320. Elsevier, MIT Press, 1990.

7. J. Gallagher. A system for specialising logic programs. Technical Report TR-91-32, Computer Science Department, University of Bristol, U.K., November 1991.

8. J. Gallagher. Specialisation of logic programs: A tutorial. In Proceedings PEPM'93, ACM SIGPLAN Symposium on Partial Evaluation and Semantics-Based Program Manipulation, pages 88-98, Copenhagen, June 1993. ACM Press.

9. J. Gallagher and M. Bruynooghe. Some low-level source transformations for logic programs. In M. Bruynooghe, editor, Proceedings Meta'90, pages 229-244, Leuven, April 1990.

10. J. Gallagher and M. Bruynooghe. The derivation of an algorithm for program specialisation. New Generation Computing, 9(3\&4):305-333, 1991.

11. J. Gallagher and D. A. de Waal. Deletion of redundant unary type predicates from logic programs. In K.-K. Lau and T. Clement, editors, Proceedings LOPSTR'92, pages 151-167. Springer-Verlag, Workshops in Computing Series, 1993.

12. P. M. Hill and J. Gallagher. Meta-programming in logic programming. Technical Report 94.22, School of Computer Studies, University of Leeds, U.K., August 1994. To appear in Volume V of the Handbook of Logic in Artificial Intelligence and Logic Programming, Oxford University Press.

13. N. D. Jones, C. K. Gomard, and P. Sestoft. Partial Evaluation and Automatic Program Generation. Prentice Hall, 1993.

14. J. Jørgensen and M. Leuschel. Efficiently generating efficient generating extensions in Prolog. In this volume.

15. J. Komorowski. An introduction to partial deduction. In A. Pettorossi, editor, Proceedings Meta'92, pages 49-69. Springer-Verlag, LNCS 649, 1992.

16. M. Leuschel. Partial evaluation of the "real thing". In F. Turini, editor, Proceedings LOPSTR'94 and META'94, pages 122-137. Springer-Verlag, Workshops in Computing Series, 1995.

17. M. Leuschel. Ecological partial deduction: Preserving characteristic trees without constraints. In M. Proietti, editor, Proceedings of LOPSTR'95. Springer-Verlag, 1996. To appear. Extended version as Technical Report CW 216, K.U.Leuven, October 1995. Accessible via http://www.cs.kuleuven.ac.be/ lpai.

18. M. Leuschel and D. De Schreye. An almost perfect abstraction operation for partial deduction using characteristic trees. Technical Report CW215, Departement Computerwetenschappen, K.U.Leuven, Belgium, October 1995. Submitted for Publication. Accessible via http://www.cs.kuleuven.ac.be/ lpai.

19. M. Leuschel and D. De Schreye. Towards creating specialised integrity checks through partial evaluation of meta-interpreters. In Proceedings of PEPM'95, the ACM Sigplan Symposium on Partial Evaluation and Semantics-Based Program Manipulation, pages 253-263, La Jolla, California, June 1995. ACM Press.

20. M. Leuschel and B. Martens. Global control for partial deduction through characteristic atoms and global trees. Technical Report CW220, Departement Computerwetenschappen, K.U.Leuven, Belgium, December 1995. Accessible via http://www.cs. kuleuven.ac.be/ lpai.

21. J. W. Lloyd. Foundations of Logic Programming. Springer-Verlag, 1987.

22. J. W. Lloyd and J. C. Shepherdson. Partial evaluation in logic programming. Journal of Logic Programming, 11(3\&4):217-242, 1991.

23. B. Martens and D. De Schreye. Automatic finite unfolding using well-founded measures. Journal of Logic Programming, 1996. To Appear, abridged and re- 
vised version of Technical Report CW180, Departement Computerwetenschappen, K.U.Leuven, October 1993, accessible via http://www.cs . kuleuven. ac. be/ Ipai.

24. B. Martens, D. De Schreye, and T. Horváth. Sound and complete partial deduction with unfolding based on well-founded measures. Theoretical Computer Science, 122(1-2):97-117, 1994.

25. B. Martens and J. Gallagher. Ensuring global termination of partial deduction while allowing flexible polyvariance. Technical Report CSTR-94-16, Computer Science Department, University of Bristol, U.K., December 1994. Accessible via http://www.cs.kuleuven.ac.be/ Ipai.

26. B. Martens and J. Gallagher. Ensuring global termination of partial deduction while allowing flexible polyvariance. In L. Sterling, editor, Proceedings ICLP'95, pages 597-611, Shonan Village Center, Kanagawa, Japan, June 1995. MIT Press.

27. T. Mogensen and A. Bondorf. Logimix: A self-applicable partial evaluator for Prolog. In K.-K. Lau and T. Clement, editors, Proceedings LOPSTR'92, pages 214-227. Springer-Verlag, Workshops in Computing Series, 1993.

28. A. Pettorossi and M. Proietti. Transformation of logic programs: Foundations and techniques. Journal of Logic Programming, 19/20:261-320, 1994.

29. D. Sahlin. Mixtus: An automatic partial evaluator for full Prolog. New Generation Computing, 12(1):7-51, 1993.

30. M. H. Sørensen and R. Glück. An algorithm of generalization in positive supercompilation. In J.W. Lloyd, editor, Proceedings ILPS'95, Portland, Oregon, December 1995. MIT Press. To Appear.

31. V. F. Turchin. The concept of a supercompiler. ACM Transactions on Programming Languages and Systems, 8(3):292-325, 1986.

32. V. F. Turchin. The algorithm of generalization in the supercompiler. In D. Bjørner, A. P. Ershov, and N. D. Jones, editors, Partial Evaluation and Mixed Computation, pages 531-549. North-Holland, 1988.

33. D. Weise, R. Conybeare, E. Ruf, and S. Seligman. Automatic online partial evaluation. In J. Hughes, editor, Proceedings of the 3rd ACM Conference on Functional Programming Languages and Computer Architecture, pages 165-191, Cambridge, Massachusets, USA, August 1991. Springer-Verlag, LNCS 523. 